\title{
Correcting the corrections for charged defects in crystals
}

\author{
Aron Walsh (iD) $1,2,3 凶$
}

While the theory of imperfections in solids is firmly established, procedures for first-principles calculations of defect quantities continue to evolve. A plethora of ad hoc correction schemes is being replaced by sophisticated self-consistent procedures that will enable more quantitative predictions of the formation energies of defect species and their spectroscopic signatures.

npj Computational Materials (2021)7:72; https://doi.org/10.1038/s41524-021-00546-0

The formation of defects in crystals is a natural consequence of chemical thermodynamics - the balance between the enthalpic cost of perturbing the atomic bonding environments and the entropic gain of introducing an ensemble of imperfections.

The key quantity that determines, in equilibrium, if a particular defect species will be abundant or rare is the free energy of formation, $\Delta G_{f}$. The concentration $\left(n_{d}\right)$ can be decomposed into contributions from enthalpy and vibrational entropy

$n_{d}=N_{\text {site }} g \exp \left(-\frac{\Delta H_{f}}{k_{\mathrm{B}} T}\right) \exp \left(\frac{\Delta S_{f}}{k_{\mathrm{B}}}\right)$

where $N_{\text {site }}$ and $g$ denote the number and degeneracy of available sites in the host crystal. The enthalpy change dominates under standard conditions, so the more burdensome vibrational term ${ }^{1}$ is most often neglected.

The first objective in defect modelling is to calculate $\Delta H_{f}$ as accurately as possible, which can be achieved in a number of ways, as illustrated in Fig. 1. Due to the exponential factor in Eq. (1), the prediction of defect concentrations is sensitive to the quality of the underlying energy terms. My brief perspective on major developments in this field is given below. I apologise in advance to the many contributions that are overlooked in this synopsis (e.g. see ref. ${ }^{2}$ for a more thorough overview).

\section{EARLY 20TH CENTURY - THEORETICAL FOUNDATIONS}

The theory of lattice dynamics developed by Born and others described the motion of atoms around their equilibrium crystallographic positions. However, what if atoms left its ideal position and wandered into an interstitial position? A classical numerical procedure for calculating the formation energy of such point defects in ionic solids was proposed by Frenkel in $1926^{3}$. The semiclassical (continuum) Mott-Littleton method for charged defects energetics was reported in $1938^{4}$, which was later expanded into a more general framework for multi-region embedded crystal calculations ${ }^{5}$. The extensive theoretical infrastructure subsequently developed for describing the electronic structure of donor and acceptor levels in semiconductors was reviewed by Pantelides in $1978^{6}$.

\section{LATE 2OTH CENTURY - PRACTICAL SOLUTIONS}

The combination of efficient algorithms to solve the Kohn-Sham equations and massively parallel computing enabled the modern era of computational materials science. Two types of approaches were developed for modelling charged point defects in crystals.

Those based on embedding potentials (a dilute defect in a host matrix as shown in Fig. 1c) offer some advantages, but remain technically challenging to set up and analyse ${ }^{7}$. A self-consistent Green's function procedure for treating defect perturbations was already reported in $1979^{\circ}$ and quickly adapted to describe the deep states associated with transition metal impurities in Si using a local density functional ${ }^{9}$.

The alternative, and more widely employed, supercell approach benefits from the robust infrastructure for calculation of crystals within periodic boundary conditions (Fig. 1d). The principal issue in describing charged defects is the electrostatic interaction between repeating centres. The long-range Coulomb interaction depends on the defect charge $q$, their spatial arrangement, and the dielectric response of the host $\epsilon(r)$. The standard solution is to introduce a homogeneous 'jellium' background charge to enforce charge neutrality in each repeat unit and ensure convergent Coulomb energy. This fix results in a shift in the average electrostatic potential and total energy of the defective supercell that must be corrected to become useful (Fig. 2).

Leslie and Gillan ${ }^{10}$ showed how to properly account for a point charge $q$ interacting with its periodic images through an isotropic dielectric medium. The approach was expanded by Makov and Payne $^{11}$ to include the quadrupole moment $(Q)$. In practice, $Q$ cannot be determined from the defect charge distribution. Defects come in many forms and their wavefunctions may be localised or delocalised and are invariably coupled to the screening host charge. In addition, the limitations of a given density functional can require further correction terms, such as valence and conduction band edge shifts, to reduce the divide between modelling and measurement ${ }^{12}$.

Exciting results and insights were gained using these methods, especially in the area of metal oxides. There were problems, however. Research groups performing similar simulations would obtain different conclusions depending on the flavour of corrections that were employed; the curious reader can look into the case of defects in $\mathrm{ZnO}$. In my opinion, the absolute defect energetics reported during this era should be taken with a pinch of salt.

\section{EARLY 21ST CENTURY-ROBUST PREDICTIONS}

To tackle these issues, a new set of correction schemes emerged for supercell calculations. Freysoldt et al. ${ }^{13}$ modelled the defect 
(a) Ideal perfect crystal

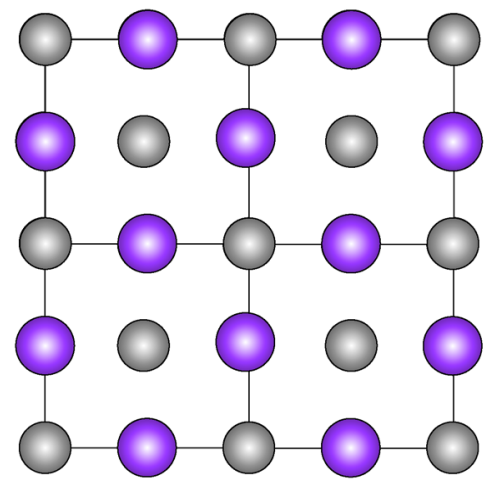

(c) Embedded cluster approach

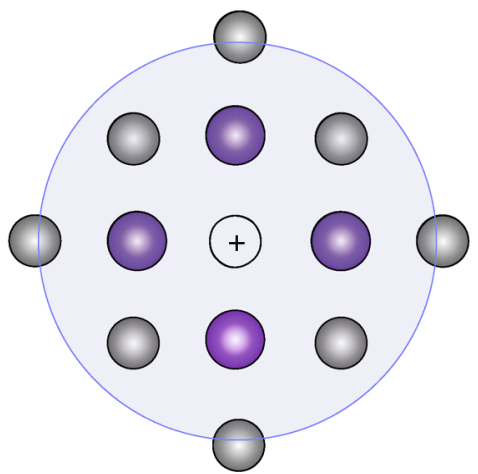

(d) Supercell approach

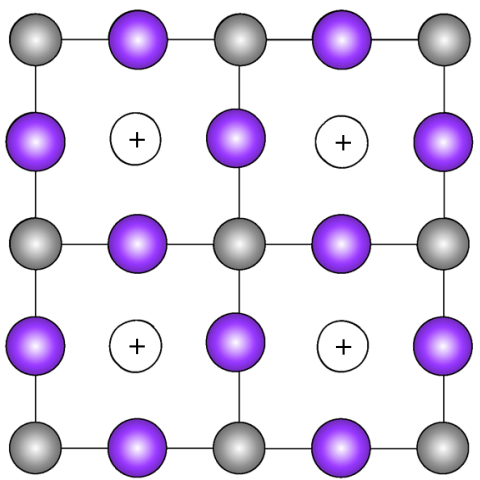

(b) Schottky disorder - charged vacancies

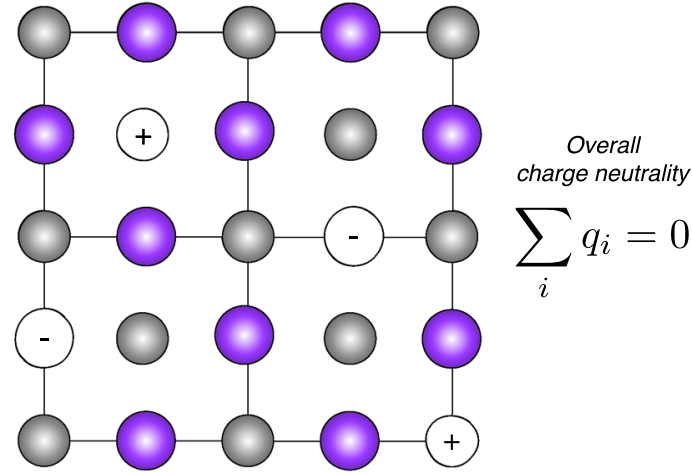

Charged defect embedded in crystal potential

Advantage:

No artificial periodic interactions

Disadvantage:

Technical complexity

$$
\begin{gathered}
\begin{array}{c}
\text { Polarisation energy } \\
\text { outside radius } r
\end{array} \\
E_{p o l}=-\frac{q^{2}}{2 r}\left(1-\frac{1}{\epsilon}\right)
\end{gathered}
$$

Charged defect repeated under periodic boundary conditions

Advantage: Technical simplicity

Leading electrostatic correction for a cubic supercell of length $L$

Disadvantage:

Artificial periodic

interactions

$$
E_{c o r r}=\frac{q^{2} \alpha}{2 \epsilon L}
$$

Fig. 1 Defect modelling. Illustration of $\mathbf{a}$ a perfect crystal and $\mathbf{b}$ a crystal containing a balanced concentration of charged vacancies. The properties of such point defects could be modelled as $\mathbf{c}$ an isolated or $\mathbf{d}$ a periodic array of charged centres in a host crystal.

charge as a Gaussian distribution in an isotropic medium. There were other efforts to include anistropic dielectric screening ${ }^{14,15}$. Kumagai and $\mathrm{Oba}^{16}$ refined these approaches with a more practical alignment procedure, based on atomic site potentials, that accounts for the full anisotropic low-frequency dielectric response.

More recent developments have moved away from a posteriori corrections of the total energy to direct modification of the underlying self-consistent calculation. The advantages are that the total energy and electronic eigenvalues can be corrected directly and more physical long-range dielectric screening can be incorporated.

A self-consistent potential correction was proposed by da Silva et al. in $2021^{17}$. The change in potential, from either a reference pristine supercell or neutral defect, is used to correct for the difference between the periodic and isolated charged defect. One limitation in the current formalism is the use of an isotropic dielectric constant; however, spatial variation is allowed in one direction for the case of slabs. In contrast, the image charge correction proposed by Suo et al. ${ }^{18}$ avoids the input of a dielectric constant at all and is instead based on the self-consistent charge density difference between charged and neutral defects, which already contains the relevant screening information. Both approaches show promising behaviour for a series of test cases including $\mathrm{NaCl}$ and $\mathrm{MgO}$.

An issue not addressed in these two recent works is how to incorporate the low-frequency dielectric response involving ion displacements. This is critical for accurately describing defect transitions, which may be excited optically or thermally ${ }^{19}$. Suo et al. $^{18}$ suggest introducing a Gaussian broadening scheme to capture ion displacements, but this remains to be implemented and tested. 


\section{Monopole}

Correction

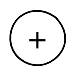

Leslie and Gillan

(1985)

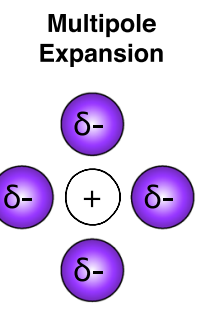

Makov and Payne (1994)
Model

Wavefunction

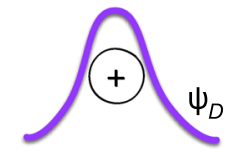

Freysoldt, Neugebauer, and Van de Walle

(2009)

\section{Self-consistent \\ Potential}

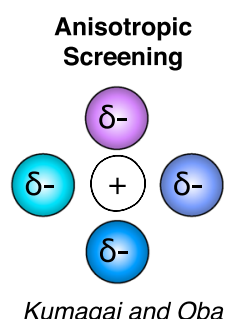

(2014)

$$
\begin{gathered}
\rho^{D^{q}}(r)-\rho^{D^{0}}(r) \\
\text { Suo et al } \\
\text { (2020) }
\end{gathered}
$$$$
V^{D^{q}}(r)-V^{B u l k}(r)
$$

da Silva et al (2021)
Fig. 2 Defect correction schemes. Illustration of the variety of correction procedures for the calculation of charged defects under periodic boundary conditions.

An entirely different approach has been taken by Xiao et al. ${ }^{20}$ in a move away from the standard jellium model to consider charge compensation by realistic valence or conduction band edge states of the host crystal. Their self-consistent correction scheme, which avoids the artificial jellium background, is intuitive for traditional semiconductors where charged defects are compensated by electrons/ holes, but less so for cases where ionic compensation dominates ${ }^{21,22}$. The approach gives good agreement with conventional methods and can also describe defects in low dimensional structures.

This new wave of research and development in the field is exciting and will further increase the predictive power of firstprinciples calculations of materials. A family of reliable and general correction schemes for charged defects will allow us to confidently tackle important scientific challenges such as equilibrium defect distributions, defect vibrations, and non-equilibrium charge transitions. They will also support more robust workflows for defect automation that can be used to identify new behaviour and physical trends in imperfect crystals.

Received: 18 March 2021; Accepted: 26 April 2021; Published online: 21 May 2021

\section{REFERENCES}

1. Gillan, M. J., Harding, J. H. \& Leslie, M. A comparison of methods for calculating defect entropies in ionic crystals. J. Phys. C 21, 5465 (1988).

2. Freysoldt, C. et al. First-principles calculations for point defects in solids. Rev. Mod. Phys. 86, 253-305 (2014).

3. Frenkel, J. Über die wärmebewegung in festen und flüssigen körpern. Z. Phys. 35, 652-669 (1926).

4. Mott, N. F. \& Littleton, M. J. Conduction in polar crystals. I. electrolytic conduction in solid salts. Trans. Farad. Soc. 34, 485-499 (1938).

5. Catlow, C. R. A. Mott-littleton calculations in solid-state chemistry and physics. J. Chem. Soc. 85, 335-340 (1989).

6. Pantelides, S. T. The electronic structure of impurities and other point defects in semiconductors. Rev. Mod. Phys. 50, 797 (1978).

7. Xie, Z. et al. Demonstration of the donor characteristics of $\mathrm{Si}$ and O defects in gan using hybrid QM/MM. Phys. Stat. Solidi (a) 214, 1600445 (2017).
8. Baraff, G. A. \& Schlüter, M. New self-consistent approach to the electronic structure of localized defects in solids. Phys. Rev. B 19, 4965-4979 (1979).

9. Zunger, A. \& Lindefelt, U. Theory of substitutional and interstitial $3 d$ impurities in silicon. Phys. Rev. B 26, 5989-5992 (1982).

10. Leslie, M. \& Gillan, M. J. The energy and elastic dipole tensor of defects in ionic crystals calculated by the supercell method. J. Phys. C 18, 973-982 (1985).

11. Makov, G. \& Payne, M. C. Periodic boundary conditions in ab initio calculations. Phys. Rev. B 51, 4014-4022 (1995).

12. Lany, S. \& Zunger, A. Assessment of correction methods for the band-gap problem and for finite-size effects in supercell defect calculations: Case studies for ZnO and GaAs. Phys. Rev. B 78, 2637-25 (2008).

13. Freysoldt, C., Neugebauer, J. \& Van de Walle, C. G. Fully ab initio finite-size corrections for charged-defect supercell calculations. Phys. Rev. Lett. 102, 016402 (2009).

14. Rurali, R. \& Cartoixà, X. Theory of defects in one-dimensional systems: application to Al-catalyzed Si nanowires. Nano Lett. 9, 975-979 (2009).

15. Murphy, S. T. \& Hine, N. D. M. Anisotropic charge screening and supercell size convergence of defect formation energies. Phys. Rev. B 87, 094111 (2013).

16. Kumagai, Y. \& Oba, F. Electrostatics-based finite-size corrections for first-principles point defect calculations. Phys. Rev. B 89, 195205 (2014).

17. da Silva, M. C. et al. Self-consistent potential correction for charged periodic systems. Phys. Rev. Lett. 126, 076401 (2021).

18. Suo, Z.-J., Luo, J.-W., Li, S.-S. \& Wang, L.-W. Image charge interaction correction in charged-defect calculations. Phys. Rev. B 102, 174110 (2020).

19. Gake, T., Kumagai, Y., Freysoldt, C. \& Oba, F. Finite-size corrections for defectinvolving vertical transitions in supercell calculations. Phys. Rev. B 101, 020102 (2020).

20. Xiao, J. et al. Realistic dimension-independent approach for charged-defect calculations in semiconductors. Phys. Rev. B 101, 165306 (2020).

21. Walsh, A., Scanlon, D. O., Chen, S., Gong, X. \& Wei, S.-H. Self-regulation mechanism for charged point defects in hybrid halide perovskites. Angew. Chem. 127, 1811-1814 (2015).

22. Walsh, A. \& Zunger, A. Instilling defect tolerance in new compounds. Nat. Mater. 16, 964-967 (2017)

\section{ACKNOWLEDGEMENTS}

I thank Seán R. Kavanagh for a careful reading of the paper. Support was received from the Faraday Institution (faraday.ac.uk; EP/S003053/1), grant No. FIRG025.

\section{AUTHOR CONTRIBUTIONS}

A.W. wrote the article.

\section{COMPETING INTERESTS}

The author declares no competing interests.

\section{ADDITIONAL INFORMATION}

Correspondence and requests for materials should be addressed to A.W.

Reprints and permission information is available at http://www.nature.com/ reprints

Publisher's note Springer Nature remains neutral with regard to jurisdictional claims in published maps and institutional affiliations.

Open Access This article is licensed under a Creative Commons Attribution 4.0 International License, which permits use, sharing, adaptation, distribution and reproduction in any medium or format, as long as you give appropriate credit to the original author(s) and the source, provide a link to the Creative Commons license, and indicate if changes were made. The images or other third party material in this article are included in the article's Creative Commons license, unless indicated otherwise in a credit line to the material. If material is not included in the article's Creative Commons license and your intended use is not permitted by statutory regulation or exceeds the permitted use, you will need to obtain permission directly from the copyright holder. To view a copy of this license, visit http://creativecommons. org/licenses/by/4.0/.

(C) The Author(s) 2021 